Prepared for the U.S. Department of Energy under Contract DE-AC05-76RL01830

\title{
Lead Slowing-Down Spectrometry Time Spectral Analysis for Spent Fuel Assay: FY11 Status Report
}
J Kulisek
C Gesh
$\mathrm{K}$ Anderson
G Warren
S Bowyer
AM Casella

September 2011

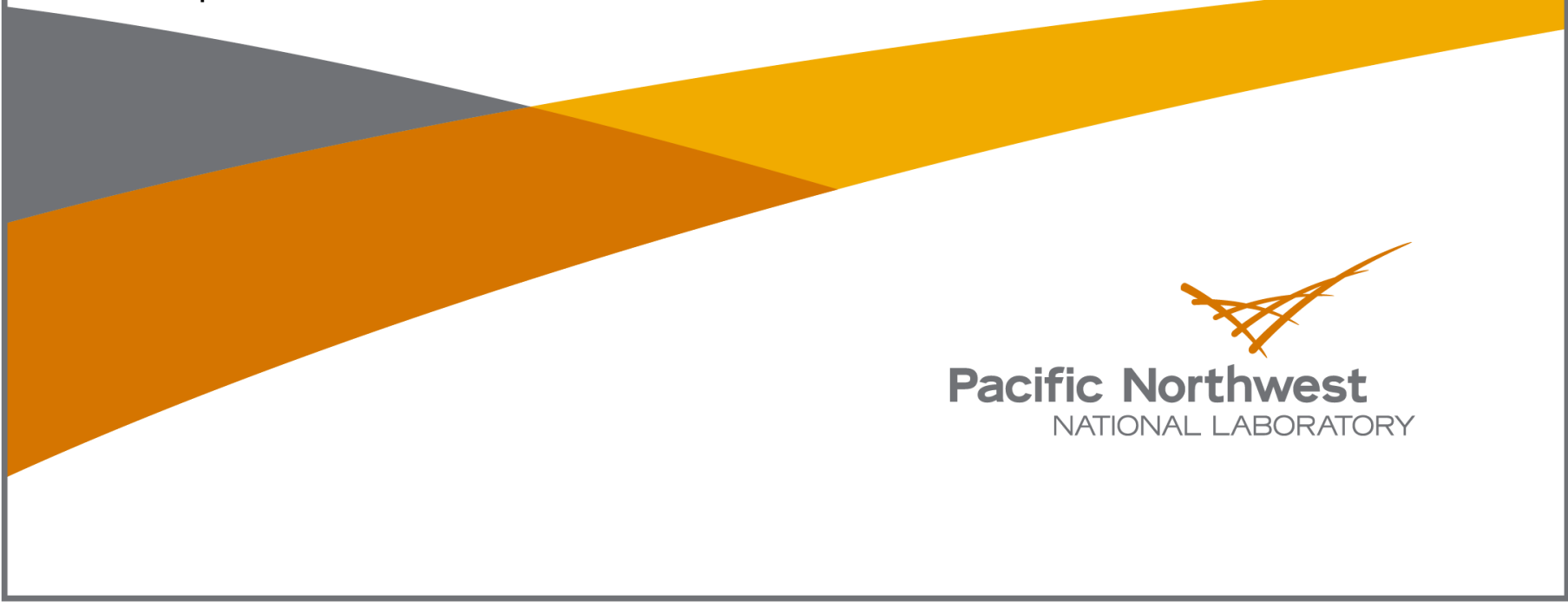




\title{
DISCLAIMER
}

This report was prepared as an account of work sponsored by an agency of the United States Government. Neither the United States Government nor any agency thereof, nor Battelle Memorial Institute, nor any of their employees, makes any warranty, express or implied, or assumes any legal liability or responsibility for the accuracy, completeness, or usefulness of any information, apparatus, product, or process disclosed, or represents that its use would not infringe privately owned rights. Reference herein to any specific commercial product, process, or service by trade name, trademark, manufacturer, or otherwise does not necessarily constitute or imply its endorsement, recommendation, or favoring by the United States Government or any agency thereof, or Battelle Memorial Institute. The views and opinions of authors expressed herein do not necessarily state or reflect those of the United States Government or any agency thereof.

\author{
PACIFIC NORTHWEST NATIONAL LABORATORY \\ operated by \\ BATTELLE \\ for the \\ UNITED STATES DEPARTMENT OF ENERGY \\ under Contract DE-AC05-76RL01830
}

Printed in the United States of America
Available to DOE and DOE contractors from the Office of Scientific and Technical Information,
P.O. Box 62, Oak Ridge, TN 37831-0062;
ph: (865) 576-8401
fax: $(865)$ 576-5728
email: reports@adonis.osti.gov

\begin{abstract}
Available to the public from the National Technical Information Service, U.S. Department of Commerce, 5285 Port Royal Rd., Springfield, VA 22161 ph: (800) 553-6847 fax: $(703) 605-6900$ email: orders@ntis.fedworld.gov online ordering: http://www.ntis.gov/ordering.htm
\end{abstract}

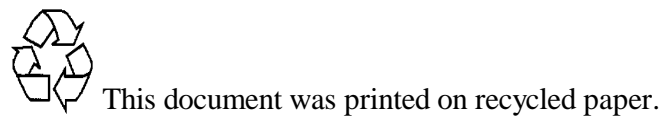




\section{Lead Slowing-Down Spectrometry Time Spectral Analysis for Spent Fuel Assay: FY11 Status Report}

J Kulisek

K Anderson

S Bowyer

AM Casella

C Gesh

G Warren

September 2011

Prepared for the U. S. DEPARTMENT OF ENERGY under Contract DE-AC05-76RL01830

Pacific Northwest National Laboratory Richland, WA 99354 
PNNL-20769 


\section{Acknowledgments}

The authors would like to thank all the project collaborators including:

Mark Shaver (PNNL) and Eric Smith (currently at the International Atomic Energy Agency).

We would also like to acknowledge the Department of Energy Fuel Cycle Research and Development Program, Material Protection, Accounting, and Control Technology (MPACT) Campaign for their support in funding this work. 


\section{Executive Summary}

Developing a method for the accurate, direct, and independent assay of the fissile isotopes in bulk materials (such as used fuel) from next-generation domestic nuclear fuel cycles is a goal of the Office of Nuclear Energy, Fuel Cycle R\&D, Material Protection and Control Technology (MPACT) Campaign. To meet this goal, MPACT supports a multi-institutional collaboration, of which PNNL is a part, to study the feasibility of Lead Slowing Down Spectroscopy (LSDS). This technique is an active nondestructive assay method that has the potential to provide independent, direct measurement of $\mathrm{Pu}$ and $\mathrm{U}$ isotopic masses in used fuel with an uncertainty considerably lower than the approximately $10 \%$ typical of today's confirmatory assay methods. This document is a progress report for FY2011 PNNL analysis and algorithm development.

Progress made by PNNL in FY2011 continues to indicate the promise of LSDS analysis and algorithms applied to used fuel. PNNL developed an empirical model based on calibration of the LSDS to simulated responses generated from well-characterized used fuel. The empirical model accounts for self-shielding effects using empirical basis vectors calculated from the singular value decomposition (SVD) of a matrix containing the true self-shielding functions of the used fuel assembly models. The potential for the direct and independent assay of the sum of the masses of ${ }^{239} \mathrm{Pu}$ and ${ }^{241} \mathrm{Pu}$ to within approximately $3 \%$ over a wide used fuel parameter space was demonstrated. PNNL also continued to develop an analytical model. Such efforts included the addition of six more non-fissile absorbers in the analytical shielding function and the nonuniformity of the neutron flux across the LSDS assay chamber. These improvements in the algorithm did not significantly improve the results of the analytical approach. A hybrid analytical-empirical approach was developed to determine the mass of total $\mathrm{Pu}$ (sum of the masses of ${ }^{239} \mathrm{Pu},{ }^{240} \mathrm{Pu}$, and ${ }^{241} \mathrm{Pu}$ ), which is an important quantity in safeguards. Results using this hybrid method were of approximately the same accuracy as the pure empirical approach. In addition, total Pu was determined with much better accuracy with the hybrid approach than the pure analytical approach.

In FY2012, PNNL will continue efforts to optimize its empirical model and minimize its reliance on calibration data. In addition, PNNL will continue to develop an analytical model, considering effects such as neutron-scattering in the fuel and cladding, as well as neutrons streaming through gaps between fuel pins in the fuel assembly. 


\section{Symbols, Acronyms and/or Initialisms}

$\begin{array}{ll}\text { GWd/MTU } & \text { Gigawatt days per metric ton of uranium } \\ \text { IAEA } & \text { International Atomic Energy Agency } \\ \text { ISU } & \text { Idaho State University } \\ \text { LANL } & \text { Los Alamos National Laboratory } \\ \text { LINAC } & \text { Linear Accelerator } \\ \text { LSDS } & \text { Lead Slowing-down Spectroscopy } \\ \text { NDA } & \text { Non-destructive assay } \\ \text { PNNL } & \text { Pacific Northwest National Laboratory } \\ \text { PWR } & \text { Pressurized water reactor } \\ \text { RPI } & \text { Rensselaer Polytechnic Institute } \\ \text { UNLV } & \text { University of Nevada, Las Vegas } \\ \text { VR } & \text { Variance reduction } \\ \varepsilon & \text { percent error: } 100 \times \mid \text { true-estimate/true }\end{array}$




\section{Contents}

$\begin{array}{lll}1.0 & \text { INTRODUCTION } & 7\end{array}$

$\begin{array}{llr}2.0 & \text { PRINCIPLES OF LSDS FOR FUEL ASSAY } & 8\end{array}$

3.0 TIME SPECTRAL ANALYSIS ALGORITHMS 9

3.1 Analytical Approach for Approximating SElf-Shielding 11

3.2 EMPIRICAl APPROACH FOR ApProXimating SElF-ShIElding 13

3.3 Hybid ANALYTICAL-EMPIRICAL APPROACH 14

4.0 RESULTS AND ANALYSIS 16

$\begin{array}{lll}5.0 & \text { FUTURE WORK } & 20\end{array}$

$\begin{array}{lll}\text { 6.0 CONCLUSIONS } & 21\end{array}$

APPENDIX A: ANALYSIS OF ANALYTICAL SELF-SHIELDING FUNCTION 22

APPENDIX B: NUMERICAL METHODS AND FITTING ALGORITHMS 27

$\begin{array}{llr}7.0 & \text { REFERENCES } & 29\end{array}$ 


\section{Figures}

Figure 2-1. Schematic of the PNNL model of a lead slowing-down spectrometer for fuel assay. The interrogating neutron population induces fission in the fuel assembly and isotopic fission chambers, and threshold fission chambers record the time-dependent production of prompt fission neutrons [6].

Figure 3-1. Example LSDS time spectra from the PNNL LSDS design: Isotope response functions, $x(t)$, for the three primary fissile isotopes of interest (left), and simulated assay signals, $y(t)$, from PWR fuels of various burnup levels (right) [6].

Figure 3-2. Ratios of the self-shielding function determined from the analytical model and the MCNP simulations. The MCNP $f(t)$ was obtained by dividing the actual average flux in the fuel by the actual average flux in the surrounding detectors as tallied in the MCNP simulations [7]. The analytical $f(t)$ was calculated using (6) for a homogenized fuel assembly.

Figure 4-1. LSDS assay results from the empirical approach for self-shielding using $4 \cdot 10^{7}$ source neutrons for the LANL 64 used PWR fuel assemblies compared with the true masses read from the MCNPX input decks. Left: ${ }^{235} \mathrm{U}$; Right: ${ }^{239} \mathrm{Pu}$ and ${ }^{241} \mathrm{Pu}$. The estimates are represented by open circles; whereas, the true values are represented by closed circles.

Figure 4-2. Calculated mass percent error, $\langle\varepsilon>$, averaged over fuel assemblies within bins having the specified number of relative assay detector counts between neutron slowingdown times of $20 \mu$ s and $2000 \mu$ s for ${ }^{235} \mathrm{U}$ (left image) and ${ }^{239} \mathrm{Pu}$ (right image). The error bars correspond to the standard deviation of the average error among assemblies within each bin of assay detector counts.

Figure 4-3. Calculated mass percent error, $\varepsilon$, versus the total true mass of the respective isotope in the used fuel assembly. 


\subsection{Introduction}

Nondestructive assay (NDA) for quantifying the amount of the individual $\mathrm{Pu}$ isotopes (i.e. ${ }^{239} \mathrm{Pu}$, ${ }^{240} \mathrm{Pu},{ }^{241} \mathrm{Pu}$ ) in used fuel assemblies is important for nuclear safeguards and used fuel storage. With respect to nuclear safeguards, it is necessary for determining shipper-receiver difference and retaining or recovering continuity of knowledge. Such measurements can also be used to support criticality calculations by verifying fuel burnup in order to maximize used fuel capacity in short-term and long-term fuel storage, and to optimize the efficient transport of used fuel.

Current NDA methods infer total Pu mass using a combination of burnup codes for calculating isotopic inventories and passive measurements of easily measured isotopes in used fuel (e.g. ${ }^{137} \mathrm{Cs}$ and $\left.{ }^{244} \mathrm{Cm}\right)$. The International Atomic Energy Agency (IAEA) has determined that such methods typically carry a Pu uncertainty of approximately $10 \%$ [1], which may result in an unaccounted $\mathrm{Pu}$ mass of more than $1000 \mathrm{~kg}$ per year in a high-volume storage or reprocessing facility.

To address these issues, this work focuses on the application of lead slowing down spectrometry (LSDS) to the measurement of isotopic masses in spent fuel. LSDS is a well-established active interrogation technique for use in nuclear cross-section measurements $[2,3]$. The goal of this effort is to use LSDS to directly measure fissile isotopes (e.g. ${ }^{235} \mathrm{U},{ }^{239} \mathrm{Pu}$, and ${ }^{241} \mathrm{Pu}$ ) in used fuel assemblies with significantly better accuracy than current NDA methods, with minimal externally provided (operator-declared) information, and in a time-efficient manner. An example of an appropriate place for LSDS in a fuel cycle would be at the receiving end of a reprocessing facility.

Earlier work by PNNL explored the use of neural networks capable of "learning" the effects of self-shielding and compensating for nonlinearities [4]. However, that work did not address the objective of truly direct measurement and required extensive calibration using well-known spent-fuel assembly standards — an expensive and in some cases, impossible, requirement. Following this, the authors explored the use of a nonlinear correction to the linear relationship using cubic splines to approximate the self-shielding effect over all slowing times [5]. The need for analyst intervention in defining knot points, and the limitations of the splines methodology to accurately track the sharp features of self-shielding, were considerable downsides to the splines approach. Subsequently, an analytical model for self-shielding was developed, which draws on tabulated nuclear data but leaves the mass of fissile and attenuating isotopes as free variables to fit [6]. The method yielded fairly reasonable assay errors within $5 \%$ over a narrow fuel parameter range and showed that LSDS was capable of full-volume assay; however, the method yielded $\sim 10 \%$ error on total $\mathrm{Pu}$ mass [7] over the entire parameter range spanned by the LANL 64 used fuel assembly library [8], as modeled in MCNPX 2.6.0 [9]. Therefore, in FY2011, in addition to continuing to study and refine its analytical model, PNNL developed an empirical model, which shows promise of significantly reducing the uncertainties of the extracted isotopic masses to below 3\% over the entire parameter range spanned by the LANL 64 used fuel 
assembly library [10]. A hybrid analytical-empirical model was also developed in order to determine the mass of ${ }^{240} \mathrm{Pu}$ in addition to the mass of the fissile isotopes, which yielded promising results.

\subsection{Principles of LSDS for Fuel Assay}

The use of LSDS for the NDA of used fuel assemblies is based on the unique resonance structure of the cross sections of the isotopes in the fuel. These resonances are strong functions of the incident neutron energy. A simplified schematic of the PNNL design of a spectrometer for use in the NDA of used nuclear fuel is shown in Figure 2-1. This figure also illustrates the process for determining the isotopic masses in the fuel assembly. The interrogating neutrons are injected into the $\mathrm{Pb}$ by a pulsed neutron source (e.g. linear accelerator (LINAC)), initially having several $\mathrm{MeV}$ of energy. Within approximately a microsecond, they lose energy via inelastic collisions with the $\mathrm{Pb}$ nuclei such that the mean interrogating neutron energy decreases to approximately $100 \mathrm{keV}$. At that time (and neutron energy), elastic scattering becomes the dominant interaction method. Then the relationship between neutron energy $E$ and slowing time $t$ is given by (1), and the energy resolution is given by (2) [11]:

$$
\begin{aligned}
E & =\frac{k}{\left(t+t_{0}\right)^{2}}, \\
\frac{\Delta E}{E} & =\sqrt{\frac{8}{3 A}} .
\end{aligned}
$$

In (1), $k$ and $t_{0}$ are parameters of the spectrometer, and $A$ is the atomic mass of the material of the spectrometer in (2). Note that the resolution is independent of the time in this approximation. The underlying assumption of (1) and (2) is that the $\mathrm{Pb}$ is completely free from impurities, particularly those having light atomic mass, such as hydrogen. Consequently, the fuel must be completely dry before being placed in the LSDS. The presence of the fuel assembly in the spectrometer interrogation chamber also has an appreciable effect on (1) and (2) [6].

As the interrogating neutrons slow down in the $\mathrm{Pb}$, they induce fissions in the various isotopes of the sample. The prompt fission neutrons emitted by these fissile isotopes contribute to the signal generated in the assay-signal sensors (e.g. fission chambers containing ${ }^{232} \mathrm{Th}$ or ${ }^{238} \mathrm{U}$ ). The fission neutrons can be distinguished from the interrogating neutrons by their energy. $\mathrm{As}^{232} \mathrm{Th}$ and ${ }^{238} \mathrm{U}$ have very low fission cross sections $(<100 \mu \mathrm{b})$ below $100 \mathrm{keV}$, they make ideal materials for assay chambers to detect the fast fission neutrons. 


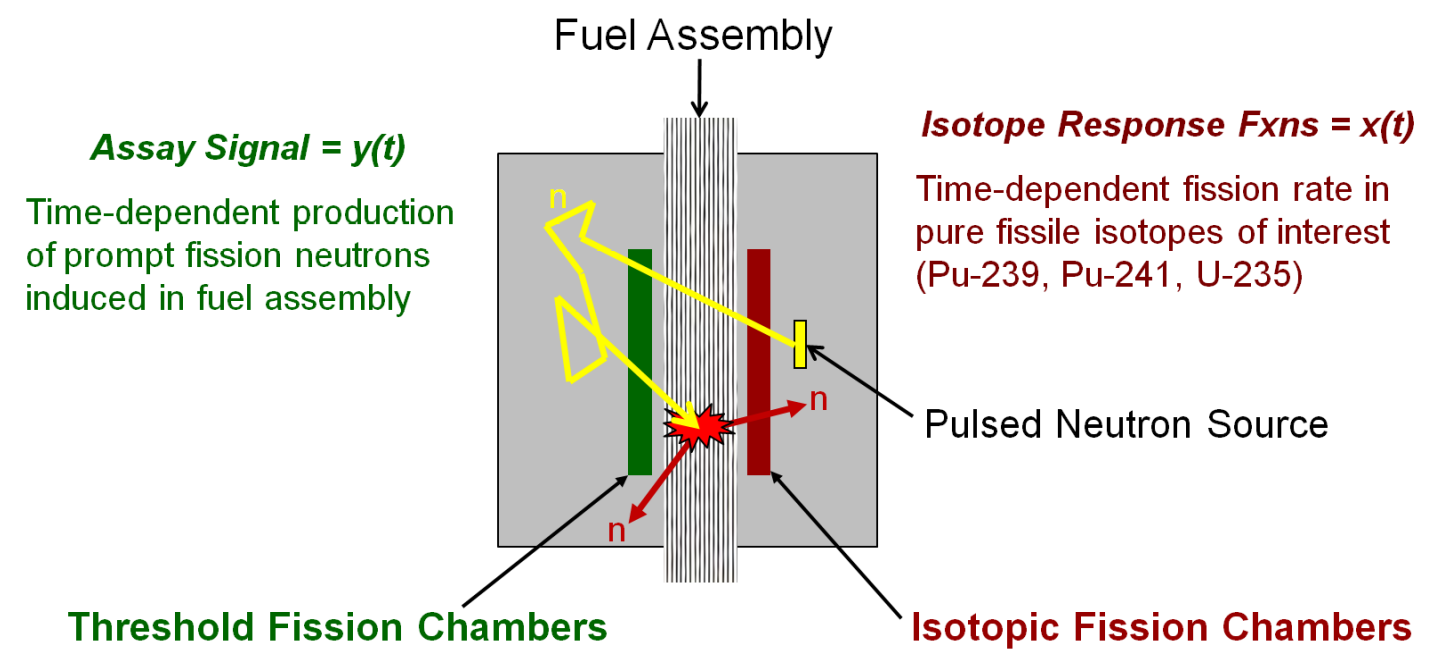

Figure 2-1. Schematic of the PNNL model of a lead slowing-down spectrometer for fuel assay. The interrogating neutron population induces fission in the fuel assembly and isotopic fission chambers, and threshold fission chambers record the time-dependent production of prompt fission neutrons [6].

Currently, no spectrometer can assay full, used fuel assemblies in order to evaluate LSDS methods described here. Therefore, this work currently relies on a modeling library of 64 fuel assemblies developed by LANL [8], which will be referred to as the LANL 64. The assemblies range in initial ${ }^{235} \mathrm{U}$ loading from $2 \%$ to $5 \%$, in burnup from 15 to $60 \mathrm{GWd} / \mathrm{MTU}$, in cooling time from 1 to 80 years. The hydrogen concentration in the fuel cladding of each assembly is set as 10 ppm per $1 \mathrm{GWd} / \mathrm{MTU}$. Variation in radial burnup within each fuel pin, as well as variation in burnup among each separate fuel pin are included within each of the 64 fuel assembly models. In MCNPX, the fuel assembly models were placed inside the assay chamber of a nominal spectrometer [6] to simulate the assay and to evaluate the mathematical models used to extract the masses of the isotopes.

\subsection{Time Spectral Analysis Algorithms}

In the PNNL approach, isotopic fission chambers measure the isotopic response to the neutron field inside the lead stack. The isotopic fission chambers are comprised of one of the isotopes of interest (e.g. ${ }^{235} \mathrm{U}$ and ${ }^{239} \mathrm{Pu}$ ) assumed to be present in the fuel assembly. The individual signals generated from induced fission in these chambers, $x_{i}(t)$, serve to deconvolve the assay signal, $y(t)$, from the threshold fission chambers (lined with either ${ }^{238} \mathrm{U}$ or ${ }^{232} \mathrm{Th}$ ), which serve to detect 
the prompt fission neutrons emitted from the fuel. Examples of simulated isotope response functions and assay signals are shown in Figure 3-1.
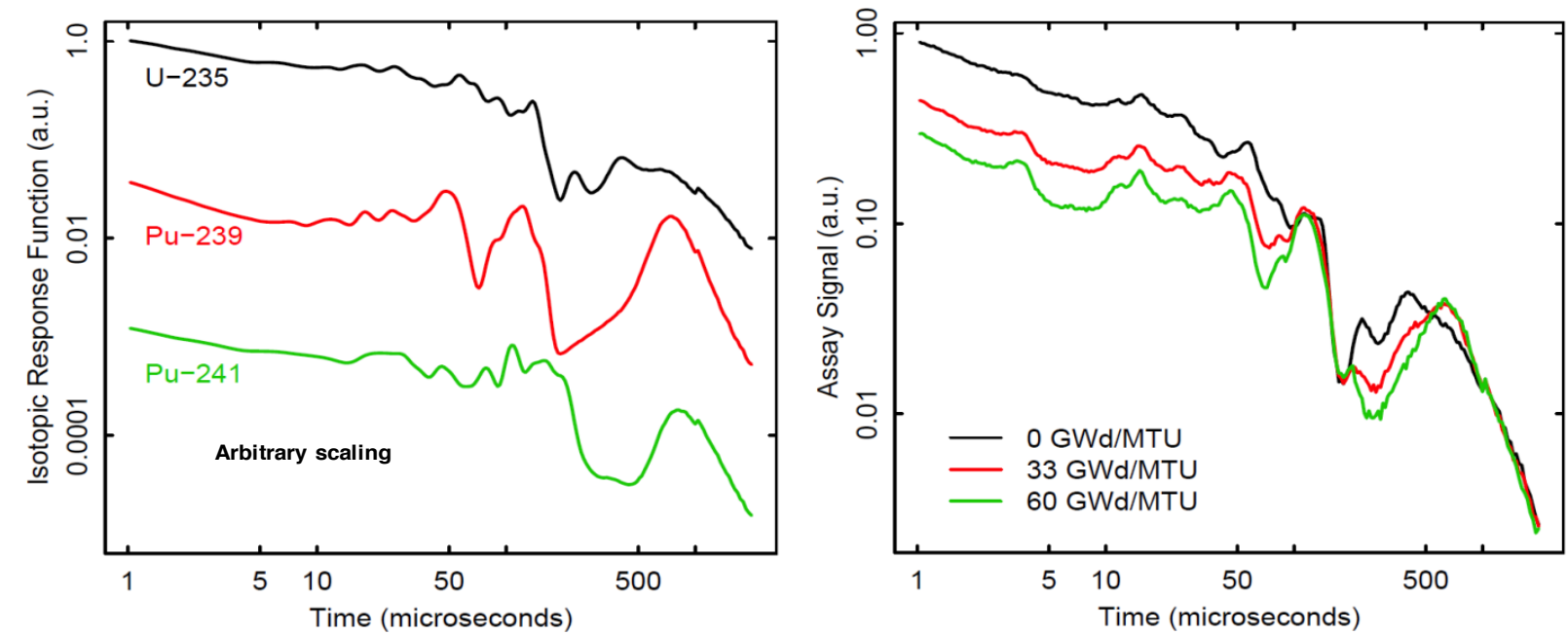

Figure 3-1. Example LSDS time spectra from the PNNL LSDS design: Isotope response functions, $x(t)$, for the three primary fissile isotopes of interest (left), and simulated assay signals, $y(t)$, from PWR fuels of various burnup levels (right) [6].

The assay signal $y(\mathrm{t})$ is expected to be a linear combination of the isotopic response functions, $x_{i}(t)$ :

$$
y(t)=C\left[a_{235} x_{235}(t)+a_{239} x_{239}(t)+a_{241} x_{241}(t)\right]=C \sum_{i} a_{i} x_{i}(t)
$$

where 235 indicates ${ }^{235} \mathrm{U}, 239$ indicates ${ }^{239} \mathrm{Pu}$ and 241 indicates ${ }^{241} \mathrm{Pu}$. In (3), it is assumed for simplicity that the efficiencies of each of the isotopic fission chambers are the same. The isotopic coefficients, $a_{i}$, are proportional to the corresponding isotopic masses, $m_{i}$, in the fuel.

One must account for the self-shielding effect caused by the presence of strong absorbers in the fuel assembly. This effect alters the interrogating neutron flux such that flux in the fuel, $\phi_{\text {fuel }}(t)$, is not equal to the flux in the fission chambers, $\phi_{\text {detectors }}(t)$. In order to account for the selfshielding, a time-dependent self-shielding function, $f(t)$, is introduced: 


$$
y(t)=f(t) C \sum_{i} \frac{m_{i} v_{i}}{A_{i}} x_{i}(t),
$$

where $v_{i}$ is the average number of neutrons released per fission. Equation (4) assumes each isotopic fission chamber contains the same number of fissile nuclei in addition to the assumptions upon which (3) is based.

The self-shielding function, $f(t)$, is defined as:

$$
f(t)=\frac{\bar{\phi}_{\text {fuel }}(t)}{\phi_{\text {detectors }}(t)} .
$$

Thus in this scheme applied by PNNL, in order to extract the isotopic masses $m_{i}$, it is necessary to measure the assay signal $y(t)$ and the isotopic responses $x_{i}(t)$ as well as have a means to determine the self-shielding function $f(t)$.

\subsection{Analytical Approach for Approximating Self-Shielding}

In FY2010, PNNL developed a first-generation analytical model for the self-shielding function $f(t)$. Using this model, one can determine the masses of fissile isotopes $\left(\right.$ e.g. ${ }^{235} \mathrm{U},{ }^{239} \mathrm{Pu}$, and ${ }^{241} \mathrm{Pu}$ ) as well as the masses of non-fissile isotopes that cause significant absorption (e.g. ${ }^{240} \mathrm{Pu}$ and ${ }^{238} \mathrm{U}$ ). While ${ }^{240} \mathrm{Pu}$ is fissile, it has a much larger $(\mathrm{n}, \gamma)$ cross section, so that it has very significant absorption effects. In this first-generation formulation, the fuel is considered as a simple, homogeneous, right parallelepiped of length $H$ and edge dimension $L$. It is assumed that the reaction cross-sections can be expressed in terms of slowing time, using the slowing-time versus energy relationship in (1). This formulation for the self-shielding function is

$$
f(t)=\frac{1-e^{-\left(\frac{2 N_{A}}{L(L+2 H)} \sum_{i} \frac{m_{i}}{A_{i}}\left(\sigma_{f}(t)+\sigma_{c}(t)\right)_{i}\right)}}{\frac{2 N_{A}}{L(L+2 H)} \sum_{i} \frac{m_{i}}{A_{i}}\left(\sigma_{f}(t)+\sigma_{c}(t)\right)_{i}},
$$

where $N_{A}$ is Avogadro's number, and $\sigma_{f}$ and $\sigma_{c}$ are the microscopic fission and capture crosssections for isotope $i$ as a function of slowing-down time [7]. The five isotopes included in the summation in the original analysis were ${ }^{235} \mathrm{U},{ }^{238} \mathrm{U},{ }^{239} \mathrm{Pu},{ }^{240} \mathrm{Pu}$, and ${ }^{241} \mathrm{Pu}$. It should be noted that (6) is slightly different than the analytical form presented in [7]. This is due to a correction in the mean chord length used of $4 V / S$ [12] as opposed to $8 V / S$ as previously reported by PNNL in [7], where $V$ is the volume and $\mathrm{S}$ is the surface area of the homogenized fuel parallelepiped. 
In FY2011, initial work at PNNL was focused on verifying and improving the implementation of $f(t)$ as formulated in (6):

- The impact of extending the selection of isotopes included in (6) was studied. Accounting for the top 11 absorbers in the self-shielding function provided little improvement compared to the original top 5 absorbers. Results are shown in Table 4-1.

- It was discovered that absorption and scattering in the ${ }^{238} \mathrm{UO}_{2}$ plays a significant role in the deviation of the flux in the fuel from the incident neutron flux that is generating the $x(t)$ signals in the isotopic fission chambers, as shown in Figure 3-2. Each used fuel assembly contains $\sim 120 \mathrm{~kg}$ of ${ }^{238} \mathrm{U}$. For the result shown in Figure 3-2, for a $3 \%$ enriched, $30 \mathrm{GWd} / \mathrm{MTU}$, 1 year cooled fuel assembly, the masses of the major five isotopes (i.e. ${ }^{235} \mathrm{U},{ }^{238} \mathrm{U},{ }^{239} \mathrm{Pu},{ }^{240} \mathrm{Pu}$ and ${ }^{241} \mathrm{Pu}$ ) were homogenized throughout the fuel assembly volume.

- The TallyX feature of MCNPX was used to calculate the actual mean chord length of the neutron paths through the assembly. This value replaced the original Dirac mean chord length in the analytical model, which was based on the assumption of isotropic and uniform flux. The calculated mean chord length differed very little from the Dirac chord length for the case when the LSDS interrogation chamber was empty (air) and when the fuel assembly contained pure ${ }^{238} \mathrm{U}$ (see Appendix A).

- The inequality of the flux in the fission chambers compared to the flux in the empty assay chamber (case with no fuel assembly) was taken into account in the model (See Appendix A). In particular, the $f(t)$ for the empty assay chamber case was found to be fairly constant at $\sim 0.8$ over the time region of interest. This was taken into account by multiplying (6) by 0.8 .

Although these modifications generally improved the agreement between the analytical $f(t)$ and the $f(t)$ obtained by MCNP simulations [7] over a significant portion of the $20 \mu \mathrm{s}$ to $2000 \mu \mathrm{s}$ slowing-down time interval, no significant improvement in the accuracy of the calculated mass estimates resulted. The first-generation $f(t)$ will need to be significantly modified if further improvements in the accuracy are to be made.

A new formulation of the self-shielding function would need to account for major deficiencies (see Appendix A). For instance, results from MCNPX simulations conducted in FY2011 indicated that the neglect of scattering within the fuel assembly and the neglect of streaming of incident neutrons between the fuel pins within the fuel lattice may contribute to discrepancies between the analytical $f(t)$ and the $f(t)$ determined directly from the MCNPX simulations. The ratio of the $f(t)$ for the analytical model and for MCNPX simulations are shown in Figure 3-2. Three cases are shown in this figure: homogenized fuel assembly containing four isotopes (excluding 238U), homogenized fuel assembly containing five isotopes (includes 238U), and a realistic geometry full fuel assembly. As shown in the figure, even for the case when the ${ }^{238} \mathrm{U}$ is included, the analytical $f(t)$ and the MCNPX-calculated $f(t)$ obtained are quite similar over the entire slowing-down time of interest. Whereas, the analytical $f(t)$ and MCNPX-calculated $f(t)$ do 
not agree as well. This indicates that neglect of streaming (by treating the fuel as homogenous) and scattering of neutrons may be the cause of the discrepancies between the analytical model and MCNPX results for $f(t)$, since accounting for the top 11 absorbers in the self-shielding function provided little improvement compared to the original top 5 absorbers.

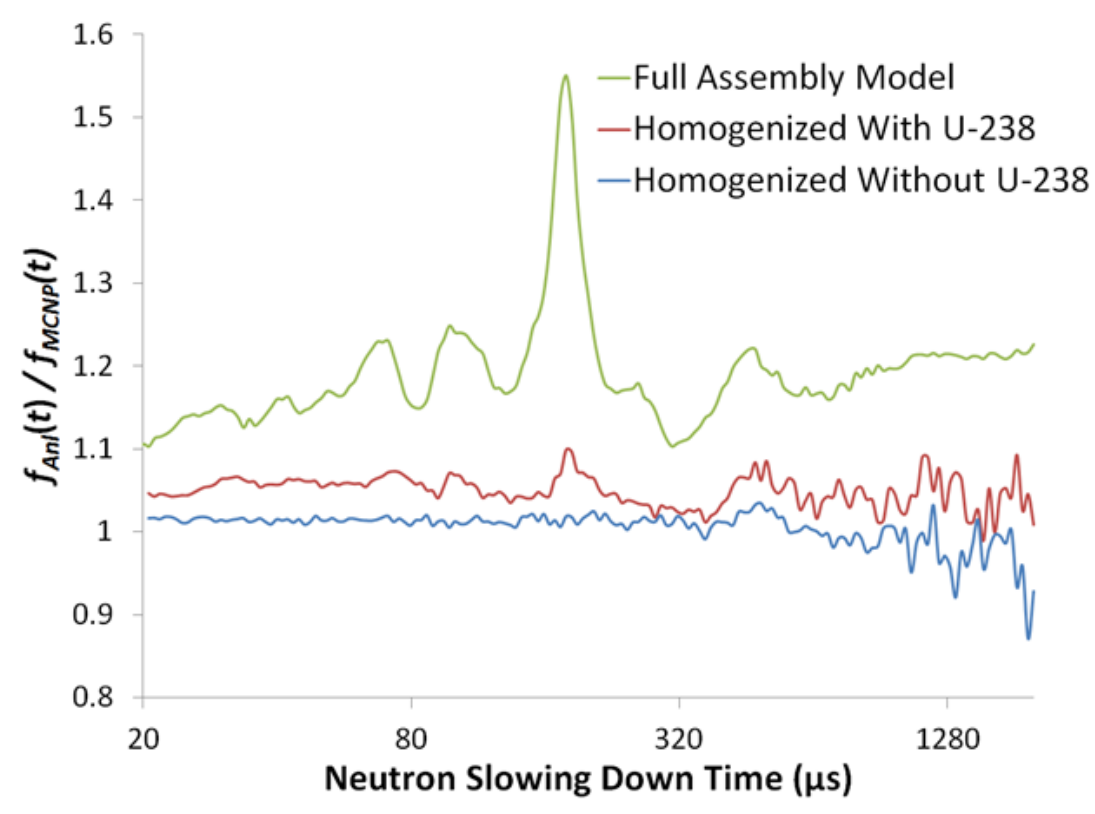

Figure 3-2. Ratios of the self-shielding function determined from the analytical model and the MCNP simulations. The MCNP $f(t)$ was obtained by dividing the actual average flux in the fuel by the actual average flux in the surrounding detectors as tallied in the MCNP simulations [7]. The analytical $f(t)$ was calculated using (6) for a homogenized fuel assembly.

\subsection{Empirical Approach for Approximating Self-Shielding}

As an alternative to the analytical approach [10], an empirical model was developed to verify that the $y(t)$ and $x(t)$ signals generated within the LSDS were sufficiently accurate to distinguish between the various assemblies within the standard LANL 64 library. This approach was based on calibration and a numerical approximation to $f(t)$ using singular value decomposition (SVD) [13]. The SVD technique reduced the data to a set of empirical basis vectors to approximate $f(t)$ 's for each of the 64 LANL fuels.

For this algorithm, the 64 true $f(t)$ 's were determined. For each of the 64 used fuel assembly models in the LANL 64, the true $m_{i}$ 's from the MCNP input decks were inserted into (4), which 
was then used to solve for $f(t)$. The $f(t)$ 's spanned 162 time bins ranging from $20 \mu \mathrm{s}$ to $2000 \mu \mathrm{s}$. A value of 1.0 was subtracted from each of these $f(t)$ 's to improve the numerical stability when performing the final step of determining the unknown masses. These 64 true $f(t)$ 's, with 1.0 subtracted, were then inserted into a matrix, $A$. The SVD of $A$ was calculated, factoring $A$ into three separate matrices:

$$
A_{64 \times 162}=U_{64 \times 64} S_{64 \times 64} V_{64 \times 162}^{T},
$$

where $U$ is a unitary matrix and $S$ is a diagonal matrix. The basis vectors $B_{i}(t)$ to be used in this analysis form the columns of $V$. The significance of this mathematical representation of $A$ is that any one of the $f(t)$ 's contained in matrix $A^{T}$ can be represented by a linear combination of the basis vectors $B_{i}(t)$. Furthermore, the diagonal matrix $S$ contains the singular values of $A$. The greater the singular value, the more closely the corresponding vector in $V$ approximates the $f(t)$ 's in $A^{T}$. From the analysis, it was determined that the five basis vectors with the largest singular values adequately describe the $f(t)$ 's; increasing the number of basis vectors did not significantly improve the results. The $f(t)$ 's were thus approximated mathematically by

$$
f(t) \approx 1.0+\sum_{j=1}^{5} b_{j} B_{j}(t),
$$

where $b_{j}$ are constants. Then, the decomposition of $f(t)$ given by (8) was substituted into (4) with the $m_{i}$ 's and $b_{j}$ 's left as unknowns. A nonlinear maximum likelihood estimation to the Poisson data generated from the MCNPX simulations was used to solve for the $m_{i}$ 's and $b_{j}$ 's (see Appendix B).

The algorithm has a practical limitation as currently implemented. All 64 fuel assembly models were used as a calibration set to determine the empirical $B_{j}(t)$ 's in (8). Conducting 64 calibration measurements, to form the basis set is impractical. Efforts are underway to evaluate the use of smaller subsets of the 64 fuel assemblies to generate the $B_{j}(t)$ 's and to understand how those smaller subsets may impact the uncertainties.

\subsection{Hybrid Analytical-Empirical Approach}

The mass of ${ }^{240} \mathrm{Pu}$, as well as the masses of other non-fissile isotopes that cause significant absorption in a used fuel assembly, cannot be determined using the empirical approach described in section 3.2. Therefore, a mixed approach making use of both the analytical and empirical approach was developed, based on the approximation

$$
f(t) \approx f_{\text {remainder }}(t) \times f_{235,239,240,241}(t),
$$


where $f_{235,239,240,241}(t)$ is the self-shielding due to ${ }^{235} \mathrm{U},{ }^{239} \mathrm{Pu},{ }^{240} \mathrm{Pu}$, and ${ }^{241} \mathrm{Pu}$, and $f_{\text {remainder }}(t)$ is the self-shielding due to other isotopes in the fuel assembly.

\section{Calibration Procedure}

As was done in the pure empirical algorithm described in section 3.2, the true values of the nonself-shielding portion of the assay signal, $y(t)$, for each of the used fuel assembly models in the LANL 64 was calculated. This was done by inserting the true mass $\left(m_{i}\right.$ 's) taken directly from the MCNP input decks for the three major fissile isotopes (i.e. ${ }^{235} \mathrm{U},{ }^{239} \mathrm{Pu}$, and ${ }^{241} \mathrm{Pu}$ ) into the summation term in the decomposition of the assay signal $y(t)$ represented by (4).

To account for the self-shielding due to ${ }^{235} \mathrm{U},{ }^{239} \mathrm{Pu},{ }^{240} \mathrm{Pu}$, and ${ }^{241} \mathrm{Pu}$, the true $m_{i}$ 's corresponding to these isotopes were inserted into the analytical self-shielding function given by (6) to calculate each of the 64 true $f_{235,239,240,241}(t)^{\prime}$ 's.

Each of the $64 y(t)$ 's was then divided by the corresponding true non-self-shielding portion of the $y(t)$ 's and also by the corresponding $f_{235,239,240,241}(t)$ to obtain the true $64 f_{\text {remainder }}(t)$ 's. According to the SVD process described in Section 3.2, these $f_{\text {remainder }}(t)$ 's were inserted into a matrix, of which the SVD was calculated. The result of this calculation, and of the calibration procedure as a whole, were eight empirical basis vectors, $B_{j}(t)$ 's, to approximate each of the $f_{\text {remainder }}(t)$ 's according to (8).

Thus, as opposed to the pure empirical algorithm described in section 3.2 where the whole selfshielding portion of the assay signal is numerically approximated using SVD, only a portion of the self-shielding is calculated using the SVD process when using the hybrid analytical-empirical approach. Also, as opposed to the pure empirical approach, eight $B_{j}(t)$ 's were required in the analytical-empirical approach rather than five in order to obtain reasonable results. As was true for the pure empirical approach, all of the 64 used fuel assemblies were used in the calibration procedure in the hybrid analytical-empirical approach.

\section{Determination of Isotopic Masses}

A nonlinear maximum likelihood estimation was then performed in order to judge whether the masses of ${ }^{235} \mathrm{U},{ }^{239} \mathrm{Pu},{ }^{240} \mathrm{Pu}$, and ${ }^{241} \mathrm{Pu}$ could be accurately determined using the $B_{j}(t)$ 's that were calculated in the calibration procedure to approximate the self-shielding effects caused by the remaining isotopes, represented by $f_{\text {remainder }}(t)$. For the nonlinear maximum likelihood estimation: the $m_{i}$ 's of ${ }^{235} \mathrm{U},{ }^{239} \mathrm{Pu},{ }^{240} \mathrm{Pu}$, and ${ }^{241} \mathrm{Pu}$ were left as unknowns in (6), for the analytical expression of $f_{235,239,240,241}(t)$; the $m_{i}$ 's corresponding to the fissile isotopes ${ }^{235} \mathrm{U},{ }^{239} \mathrm{Pu}$, ${ }^{241} \mathrm{Pu}$ were left as unknowns in the non-self-shielding summation term in (4); and the $b_{j}(\mathrm{t})$ 's were left as unknowns in the numerical approximation of $f_{\text {remainder }}(t)$ given by $(8)$. A nonlinear maximum likelihood estimation was then performed to determine the $m_{i}$ 's and $b_{j}(\mathrm{t})$ 's, as described in Appendix B. 


\subsection{Results and Analysis}

Results using the empirical approach applied to the LANL 64 assemblies are shown in Figure 4-1. The figure compares the masses for ${ }^{235} \mathrm{U},{ }^{239} \mathrm{Pu}$, and ${ }^{241} \mathrm{Pu}$ as estimated from the algorithm versus the true masses for each of the LANL 64 used fuel assemblies. Previous results had been shown recently in [10]. Those results were based on throwing $10^{7}$ source neutrons; note that variation reduction (VR) techniques enable a significant reduction of thrown neutrons in the simulation compared to physical neutrons. Time-dependent weight-window VR was used in this work [7]. The results shown in Figure 4-1 were generated with four times as many source neutrons as in [10]. The average percent error, $<_{\varepsilon}>$, as well as the maximum percent error, $\varepsilon_{m}$, over each set of 16 fuels having the same level of burnup, is also shown. For $\mathrm{Pu},<_{\varepsilon}>$ and $\varepsilon_{m}$ are given for the sum of ${ }^{239} \mathrm{Pu}$ and ${ }^{241} \mathrm{Pu}$. The results show significant improvement over previous results using the analytical model [7].

A side-by-side comparison is presented in Table 4-1 among the results obtained using:

- the empirical algorithm with $4 \cdot 10^{7}$ source neutrons (Figure 4-1)

- the same empirical algorithm but with $1 \cdot 10^{7}$ source neutrons [10]

- the first generation analytical algorithm with 5 isotopes [7]

- the analytical model with 11 isotopes [10]

- the hybrid analytical-empirical approach described in section 3.3 of this paper

From this table, one can see that the increase in the number of thrown neutrons significantly reduces both the average percent error and maximum percent error. This result suggests that some of the difference between modeled fuel isotopic masses and extracted isotopic masses was due in part to poor statistics in the simulation. In addition, it is clear that the empirical approach, even with the poorer statistics, provides better results than the current analytical approach. In addition, with the exception of the ${ }^{235} \mathrm{U}$ mass for $60 \mathrm{GWd} / \mathrm{MTU}$, all of the higher statistical results for the empirical model have average percent errors less than $1.5 \%$. The results in terms of average and maximum percent error for the hybrid analytical-empirical approach are approximately the same as the empirical approach for the same number of thrown neutrons. In addition, the average and maximum percent error are significantly lower than the analytical approaches with either 5 or 11 isotopes for total Pu mass, an important quantity in international safeguards. The ${ }^{240} \mathrm{Pu}$ mass cannot be determined using the pure empirical approach. 

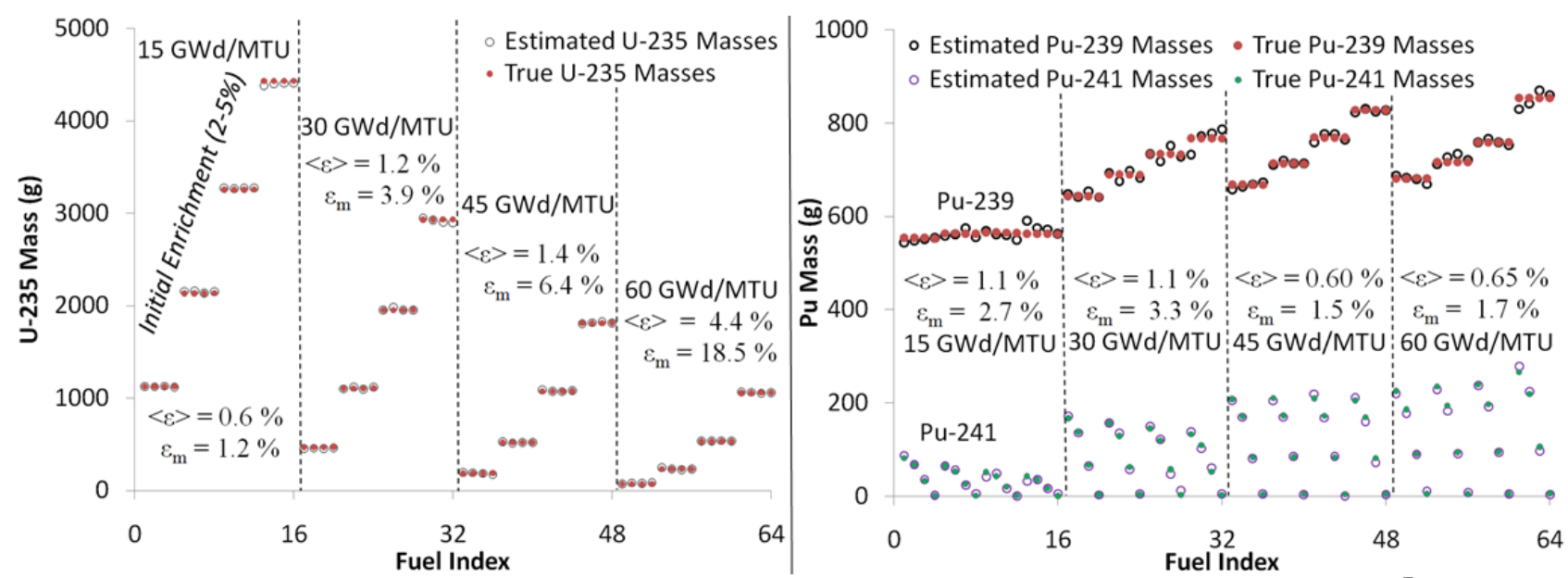

Figure 4-1. LSDS assay results from the empirical approach for self-shielding using $4 \cdot 10^{7}$ source neutrons for the LANL 64 used PWR fuel assemblies compared with the true masses read from the MCNPX input decks. Left: ${ }^{235} \mathrm{U}$; Right: ${ }^{239} \mathrm{Pu}$ and ${ }^{241} \mathrm{Pu}$. The estimates are represented by open circles; whereas, the true values are represented by closed circles.

Table 4-1. Average percent error $\langle\varepsilon\rangle$ and maximum percent error $\varepsilon_{\mathrm{m}}$ grouped by burnup for the results presented for the empirical algorithm using $4 \cdot 10^{7}$ source neutrons (Figure 4-1), the empirical algorithm with $10^{7}$ source neutrons [10], and the analytical model with $10^{7}$ source neutrons and 5 isotopes [7], the analytical model with $10^{7}$ source neutrons and 11 isotopes [10], and the hybrid analytical-empirical approach described in section 3.3 of this paper.

\begin{tabular}{|c|c|c|c|c|c|c|}
\hline Isotope & Method & $\begin{array}{c}15 \\
\mathrm{GWd} / \mathrm{MTU} \\
<_{\varepsilon}>\mid \varepsilon_{\mathrm{m}}(\%) \\
\end{array}$ & $\begin{array}{c}30 \\
\text { GWd/MTU } \\
<\varepsilon>\mid \varepsilon_{\mathrm{m}}(\%) \\
\end{array}$ & $\begin{array}{c}45 \\
\text { GWd/MTU } \\
<\varepsilon>\mid \varepsilon_{\mathrm{m}}(\%) \\
\end{array}$ & $\begin{array}{c}60 \\
\text { GWd/MTU } \\
<\varepsilon>\mid \varepsilon_{\mathrm{m}}(\%) \\
\end{array}$ & $\begin{array}{c}\text { Overall } \\
<\varepsilon>\mid \varepsilon_{\mathrm{m}}(\%) \\
\end{array}$ \\
\hline \multirow[t]{5}{*}{${ }^{235} \mathrm{U}$} & Empirical $\left(4 \cdot 10^{7}\right)$ & $\begin{array}{l}0.60 \mid 1.2 \\
\end{array}$ & $1.2 \mid 3.9$ & $1.4 \mid 6.4$ & $4.4 \mid 18$ & $1.9 \mid 18$ \\
\hline & Empirical $\left(10^{7}\right)$ & \begin{tabular}{l|l}
3.3 & 11 \\
\end{tabular} & \begin{tabular}{l|l|l}
3.7 & 16 \\
\end{tabular} & \begin{tabular}{l|l|l}
4.3 & 8.7 \\
\end{tabular} & $6.7 \mid 22$ & \begin{tabular}{l|l}
4.5 & 22 \\
\end{tabular} \\
\hline & Analytical (5 iso) & \begin{tabular}{l|l}
11 & 19 \\
\end{tabular} & \begin{tabular}{l|l|l}
13 & 31 \\
\end{tabular} & \begin{tabular}{l|l}
39 & 120 \\
\end{tabular} & \begin{tabular}{l|l|l}
125 & 390 \\
\end{tabular} & \begin{tabular}{l|l|}
47 & 390 \\
\end{tabular} \\
\hline & Analytical (11 iso) & \begin{tabular}{l|l|}
9.2 & 19 \\
\end{tabular} & \begin{tabular}{l|l|}
12 & 37 \\
\end{tabular} & \begin{tabular}{l|l}
31 & 88 \\
\end{tabular} & \begin{tabular}{l|l}
82 & 230 \\
\end{tabular} & $34 \mid 230$ \\
\hline & Hybrid $\left(4 \cdot 10^{7}\right)$ & \begin{tabular}{l|l|l|}
0.66 & 1.7 \\
\end{tabular} & \begin{tabular}{l|l|l|}
1.3 & 3.2 \\
\end{tabular} & \begin{tabular}{l|l|l}
1.6 & 6.7 \\
\end{tabular} & $3.0 \mid 12$ & \begin{tabular}{l|l}
1.6 & 12 \\
\end{tabular} \\
\hline \multirow{5}{*}{$\begin{array}{l}{ }^{239} \mathrm{Pu}+ \\
{ }^{241} \mathrm{Pu}\end{array}$} & Empirical $\left(4 \cdot 10^{7}\right)$ & $1.1 \mid 2.7$ & \begin{tabular}{ll|l|}
1.1 & 3.3 \\
\end{tabular} & $0.60 \mid 1.5$ & $0.65 \mid 1.7$ & $\begin{array}{l}0.89 \mid 3.3 \\
\end{array}$ \\
\hline & Empirical $\left(10^{7}\right)$ & \begin{tabular}{l|l|l}
2.8 & 7.5 \\
\end{tabular} & \begin{tabular}{l|l|}
2.4 & 7.7 \\
\end{tabular} & $3.8 \mid 11$ & $5.8 \mid 21$ & $3.7 \mid 21$ \\
\hline & Analytical (5 iso) & \begin{tabular}{l|l|}
4.0 & 11 \\
\end{tabular} & \begin{tabular}{l|l}
4.4 & 12 \\
\end{tabular} & \begin{tabular}{ll|l}
$4.4 \mid 11$ \\
\end{tabular} & $4.5 \mid 12$ & $4.3 \mid 12$ \\
\hline & Analytical (11 iso) & $9.1 \mid 20$ & \begin{tabular}{l|l|l}
6.6 & 14 \\
\end{tabular} & \begin{tabular}{l|l}
8.5 & 17 \\
\end{tabular} & \begin{tabular}{l|l|}
10 & 23 \\
\end{tabular} & \begin{tabular}{l|l|l}
8.6 & 23 \\
\end{tabular} \\
\hline & Hybrid $\left(4 \cdot 10^{7}\right)$ & \begin{tabular}{l|l|l}
1.8 & 6.7 \\
\end{tabular} & \begin{tabular}{l|l|l|}
1.2 & 2.2 \\
\end{tabular} & $0.73 \mid 1.5$ & $0.58 \mid 2.0$ & \begin{tabular}{l|l|l}
1.1 & 6.7 \\
\end{tabular} \\
\hline \multirow{3}{*}{$\begin{array}{l}{ }^{239} \mathrm{Pu}+ \\
{ }^{240} \mathrm{Pu}+ \\
{ }^{241} \mathrm{Pu}\end{array}$} & Analytical (5 iso) & \begin{tabular}{l|l}
12 & 22 \\
\end{tabular} & \begin{tabular}{l|l}
5.9 & 15
\end{tabular} & \begin{tabular}{l|l|l}
3.5 & 9.4 \\
\end{tabular} & $7.4 \mid 16$ & $7.2 \mid 22$ \\
\hline & Analytical (11 iso) & \begin{tabular}{l|l}
15 & 26 \\
\end{tabular} & $7.7 \mid 19$ & $7.8 \mid 21$ & \begin{tabular}{l|l|l}
9.8 & 18 \\
\end{tabular} & $10 \mid 26$ \\
\hline & Hybrid $\left(4 \cdot 10^{7}\right)$ & \begin{tabular}{l|l|l}
3.0 & 8.4 \\
\end{tabular} & $2.0 \mid 3.7$ & \begin{tabular}{l|l|l|}
1.2 & 3.9 \\
\end{tabular} & $\begin{array}{l}0.96 \mid 2.3 \\
\end{array}$ & \begin{tabular}{l|l|}
1.8 & 8.4 \\
\end{tabular} \\
\hline
\end{tabular}


An analysis was performed in order to determine whether running the MCNP simulations with additional particles would improve upon the average errors reported above. In addition, the results of the uncertainty analysis were used to determine the number of neutrons that would be required in practice to achieve the accuracy on which the calculated mass estimates shown in Figure 4-1 were based. This analysis is discussed below.

Results illustrating the average percent error for the ${ }^{235} \mathrm{U}$ and ${ }^{239} \mathrm{Pu}$ mass estimates versus average number of counts tallied in the ${ }^{238} \mathrm{U}$ assay fission chamber detectors are shown in Figure 4-2. The neutron slowing-down times were limited to between $20 \mu \mathrm{s}$ and $2000 \mu \mathrm{s}$. The average percent errors for the ${ }^{235} \mathrm{U}$ mass estimates were much larger for the cases where fewer counts were detected in the assay chambers. In contrast, the average percent errors for the ${ }^{239} \mathrm{Pu}$ mass estimates were relatively insensitive to the average number of tallied counts in the assay detectors.
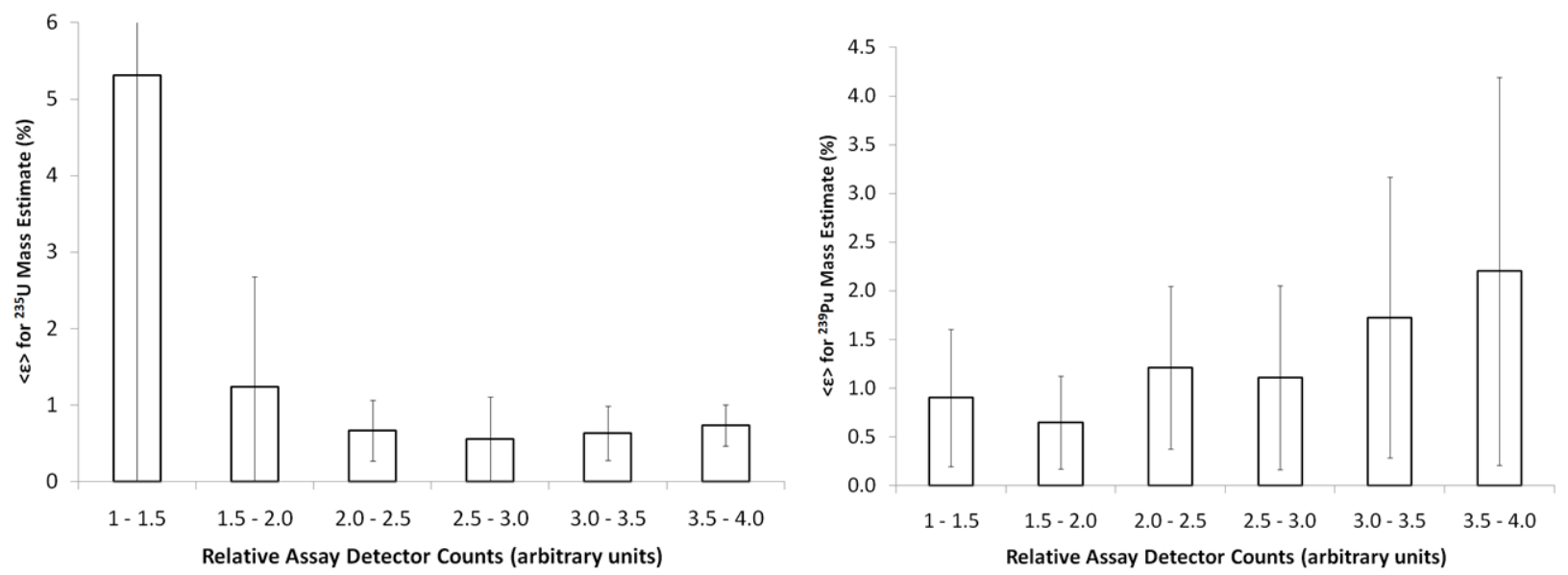

Figure 4-2. Calculated mass percent error, $\langle\varepsilon>$, averaged over fuel assemblies within bins having the specified number of relative assay detector counts between neutron slowing-down times of $20 \mu \mathrm{s}$ and $2000 \mu \mathrm{s}$ for ${ }^{235} \mathrm{U}$ (left image) and ${ }^{239} \mathrm{Pu}$ (right image). The error bars correspond to the standard deviation of the average error among assemblies within each bin of assay detector counts.

Additional analysis was performed comparing the percent error for the calculated mass estimates versus the total mass of the respective isotope present in the used fuel assembly. The results of this analysis are shown together in Figure 4-3 for ${ }^{235} \mathrm{U},{ }^{239} \mathrm{Pu},{ }^{241} \mathrm{Pu}$, and the sum of the masses of all three fissile isotopes. The largest percent errors for ${ }^{235} \mathrm{U}$ were obtained for the fuel assemblies containing the lowest total mass of ${ }^{235} \mathrm{U}$ present in the entire fuel assembly. For three out of the four assemblies containing less than $100 \mathrm{~g}$ of ${ }^{235} \mathrm{U}$, the calculated ${ }^{235} \mathrm{U}$ mass differed from the true mass by more than $10 \%$. The percent error for the ${ }^{239} \mathrm{Pu}$ calculated estimates was fairly 
insensitive to the total mass of ${ }^{239} \mathrm{Pu}$ in the fuel assembly. This may be a consequence of all of the fuel assemblies containing ${ }^{239} \mathrm{Pu}$ masses greater than $500 \mathrm{~g}$ and of smaller variations in the mass of ${ }^{239} \mathrm{Pu}$ across the LANL 64 used fuel assemblies. For ${ }^{241} \mathrm{Pu}$, significantly larger percent errors were obtained for the calculated mass estimates, particularly for masses less than $10 \mathrm{~g}$.
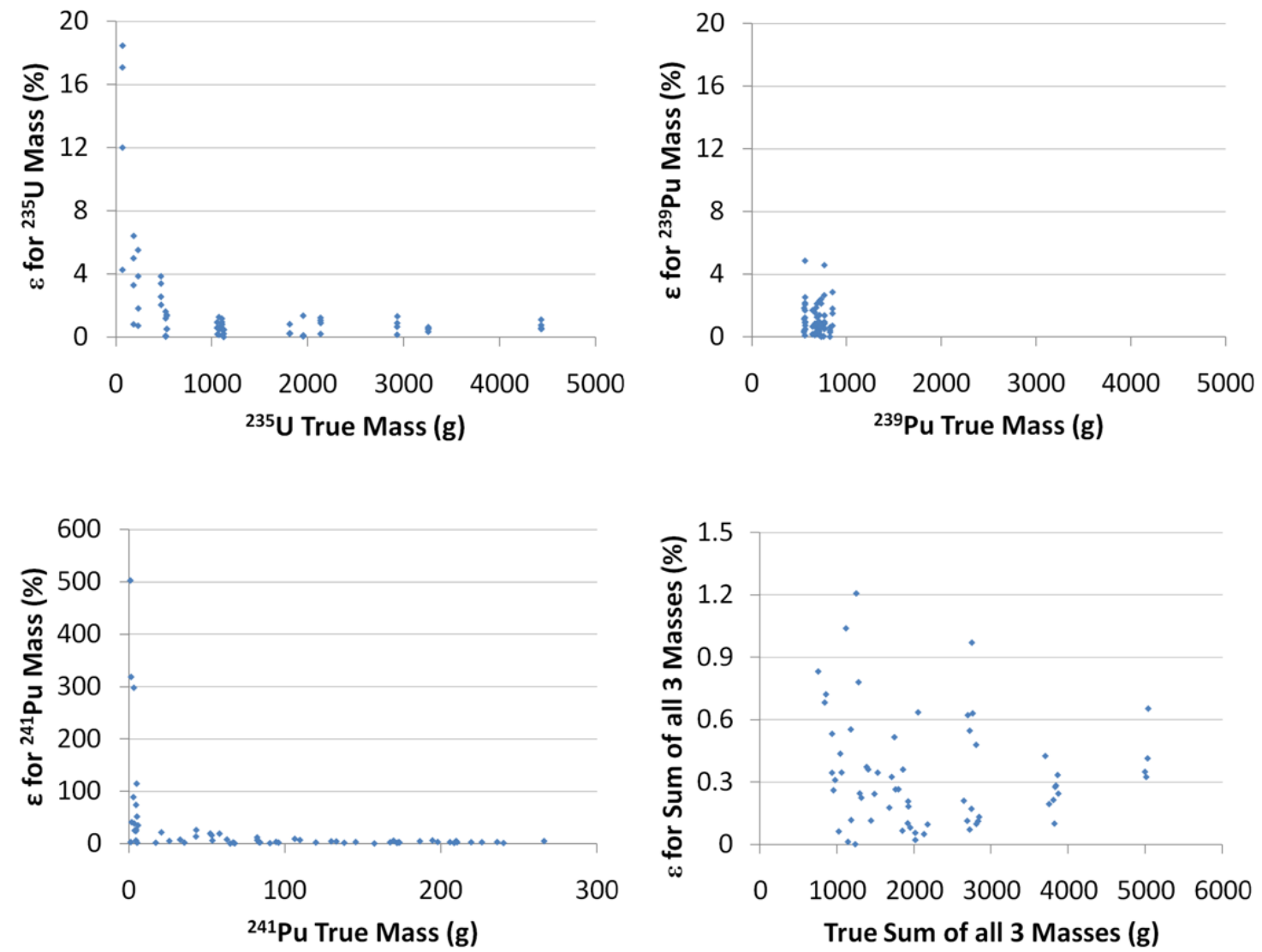

Figure 4-3. Calculated mass percent error, $\varepsilon$, versus the total true mass of the respective isotope in the used fuel assembly.

In addition, three of the 64 fuels were simulated without VR. In the $20 \mu$ s to $2000 \mu$ s neutron slowing-down region, the fractional errors associated with the tallied counts in the ${ }^{238} \mathrm{U}$ assay detector for these three simulations were then compared to fractional errors for the MCNP simulations using VR for the same three fuels. From this comparison, the total number of source neutrons required, without using VR, to achieve the same level of statistical precision as that without using VR, across all time bins in the slowing-down region of interest, was determined. To determine the total number of source neutrons required in an actual spectrometer of the same design that was modeled in the simulations, the calculated result for the total number of source neutrons required without using VR was multiplied by an assumed absolute detection efficiency 
of $0.1 \%$ for the $32{ }^{238} \mathrm{U}$ assay detectors combined, as estimated in a previous report [7]. The results of this calculation are given in Table 4-2. From these preliminary results, the original assumption in [7] of a total of $10^{16}$ source neutrons required for an assay of a used fuel assembly is reasonable, and is similar to the LANL conclusions [14]. Such an assay can theoretically be completed comfortably within an hour with the use of a LINAC as a pulsed neutron source.

Table 4-2. Number of source neutrons required in an experiment to achieve the same statistical precision across all time bins in the ${ }^{238} \mathrm{U}$ assay signal as the results upon which the calculated mass estimates presented in this report were based.

\begin{tabular}{|c|c|c|c|c|c|c|}
\hline $\begin{array}{l}\text { Fuel } \\
\text { Index } \\
(\#)\end{array}$ & $\begin{array}{l}\text { Burnup } \\
(\mathrm{GWd} / \mathrm{MTU})\end{array}$ & $\begin{array}{l}\text { Initial } \\
\text { Enrichment } \\
(\%)\end{array}$ & $\begin{array}{l}\text { Cooling } \\
\text { Time } \\
(\mathrm{yr})\end{array}$ & $\begin{array}{l}\text { MCNP } \\
\text { Source } \\
\text { Neutrons } \\
\text { (Using VR) }\end{array}$ & $\begin{array}{l}\text { MCNP } \\
\text { Scaled } \\
\text { Source } \\
\text { Neutrons } \\
\text { (No VR) }\end{array}$ & $\begin{array}{l}\text { Actual LSDS } \\
\text { Required } \\
\text { Source } \\
\text { Neutrons }\end{array}$ \\
\hline 23 & 30 & 3 & 20 & \multirow{3}{*}{$4 \cdot 10^{7}$} & $1.4 \cdot 10^{12}$ & $1.4 \cdot 10^{15}$ \\
\hline 36 & 45 & 2 & 80 & & $1.6 \cdot 10^{12}$ & $1.6 \cdot 10^{15}$ \\
\hline 52 & 60 & 2 & 80 & & $1.9 \cdot 10^{12}$ & $1.9 \cdot 10^{15}$ \\
\hline
\end{tabular}

\subsection{FUTURE WORK}

Further study is needed to test and develop confidence in the calibration-based algorithm. Therefore, the algorithm will be tested with a subset of the LANL 64 used fuel assemblies to generate the calibration data for numerically estimating the time-dependent self-shielding functions. In addition, the algorithm will be tested on models of fuels having different initial enrichments, burnups and/or cooling times than those of the LANL 64 used fuel assemblies.

The first generation analytical model will be modified in an attempt to accurately account for the streaming, scattering, and absorption that occur within the fuel assemblies and are not appropriately accounted for at present. This modification will proceed by a close inspection of the neutron physics occurring due to simplified interrogation targets, such as single pins and pin arrays representing sections of assemblies. This procedure will provide insight into the quantification of the importance of streaming, scattering and absorption in the fuel assemblies. A new semi-empirical model will be generated from these studies that will in turn be used to gain insight into the generation of a new fully analytical model.

The incorporation of additional information will be investigated. These may include estimates of burnup from measurements using ${ }^{134} \mathrm{Cs} /{ }^{137} \mathrm{Cs}$ gamma-ray peak ratios or limited operatorprovided information, which if incorrect, would cause the results to significantly diverge. Such 
information can be incorporated into the calibration algorithm by enabling one to bias the selection of fuels used in the calibration set.

Future efforts must also include the benchmarking of the calibration algorithm with the experimental data from actual LSDS instruments, such as those available at the LANL LANSCE facility and the Gaerttner LINAC facility at RPI. For example, the calibration algorithm can be applied to the RPI fresh fuel pin measurements that were conducted in FY2011 by using MCNP to generate calibration data by simulating the assay of a single fuel pin and varying its isotopic fuel composition.

\subsection{CONCLUSIONS}

The potential for LSDS to provide direct, independent, and accurate assay of $U$ and Pu isotopes in used fuel relies heavily on developing time-spectra analysis methods that can account for the nonlinear effects of self-shielding and neutron absorption by non-fissile isotopes. The timespectra analysis method development efforts from FY2011 were evaluated using a (LANLdeveloped) simulated library of PWR used fuel assemblies that spans a wide range of initial enrichment, burnup, cooling time (and included pin-to-pin and within-pin burnup variation). The major conclusions of the FY2011 evaluation of the time spectral analysis algorithm are summarized below:

For the wide parameter space spanned by the LANL 64 used fuel assemblies, the calibrationbased SVD algorithm allows the direct and independent assay of ${ }^{239} \mathrm{Pu}$ and ${ }^{241} \mathrm{Pu}$ to an accuracy within approximately 3\%. Using all 64 LANL fuels in the calibration to generate five empirical basis vectors, the accuracy for the sum of the masses of ${ }^{239} \mathrm{Pu}$ and ${ }^{241} \mathrm{Pu}$ was determined within $3.3 \%$ for each of the LANL 64 fuel assemblies. For $90 \%$ of the assemblies, this sum was calculated to within $2 \%$ accuracy, and for $65 \%$ of the assemblies, the accuracy was determined to within $1 \%$. The accuracy of this calibration method now appears to be limited by the overall sensitivity of the LSDS in detecting small total amounts of fissile isotopes present in the fuel assemblies.

Significant changes to the first generation analytical model are necessary in order to account for the scattering and streaming within the fuel assembly. Insertion of the cross-sections of additional absorbers into Eq. (6) presented little benefit and investigation of the neutron paths across the assemblies demonstrated the necessity to develop a new analytical form for $f(t)$. 


\section{Appendix A: Analysis of Analytical Self-Shielding Function}

This derivation and analysis of the analytical $f(t)$ given in this appendix are given as an alternative to the derivation given in [7] with the intent to more clearly illustrate the various assumptions on which the analytical $f(t)$ is based. The number of neutron absorptions, $A$, per unit volume and per unit time occurring within a volume containing a homogeneous mixture of neutron-absorbing material (e.g. fuel), is

$$
A=\Sigma_{A} \bar{\phi}_{\text {fuel }}(t)
$$

$\Sigma_{A}$ is the total macroscopic absorption cross section, defined as the probability per unit path

length that a neutron passing through the fuel volume is absorbed. $\bar{\phi}_{\text {fuel }}(t)$ is the volumeaveraged neutron fluence rate (i.e. flux) within the fuel, which is defined as the sum of all neutron path lengths per unit volume and per unit time.

However, $A$ can also be derived considering exponential attenuation. That is, the probability that a neutron is absorbed along an average, straight chord length of $r$, assuming that the neutron will not scatter, that the neutron enters from outside the volume (i.e. not produced within the volume), and that the material is homogenous within the volume is

$$
1-e^{-\Sigma_{A} r}
$$

Therefore, the total number of neutrons absorbed within the fuel, per unit volume, is also given by (A.3), where $N(t)$ is the total number of neutrons entering the fuel volume per unit time, and $V$ is the size of the fuel volume:

$$
A \approx \frac{\left(1-e^{-\Sigma_{A} r}\right) \times N(t)}{V} .
$$

Equating (A.1) and (A.3) and solving for $\bar{\phi}_{f u e l}(t)$ results in

$$
\bar{\phi}_{f u e l}(t) \approx \frac{\left(1-e^{-\Sigma_{A} r}\right) \times N(t)}{\Sigma_{A} V} .
$$

Considering the PNNL LSDS model described in section 2.0, substitution of (A.4) into (5) yields 


$$
f(t) \approx \frac{\left(1-e^{-\Sigma_{A} r}\right) \times N(t)}{\Sigma_{A} V \phi_{\text {detectors }}(t)} .
$$

Consider the case when the interrogation chamber contains a material (e.g. air) for which neutron interactions can be assumed to be negligible, such that $\phi_{\text {detectors }}(t)$ equals the flux in the empty volume in the interrogation chamber that would be occupied by the fuel assembly when full. Then, $\phi_{\text {detectors }}(t)$ could be calculated is

$$
\phi_{\text {detectors }}(t)=\frac{N(t) r}{V}
$$

where, again, $V$ is the volume of the space occupied by the fuel assembly when present in the interrogation chamber, and $r$ is the mean chord length of neutrons through this volume. The neutron flux is simply defined as the total neutron path length per unit time and per unit volume. Any absorption within the volume would decrease the neutron path length below $r$. Substitution of (A.6) into (A.5) yields

$$
f(t)=\frac{\left(1-e^{-\Sigma_{A} r}\right)}{\Sigma_{A} r} .
$$

If one considers a right parallelepiped fuel volume with length $H$ and edge $L$, such that the volume is given by $V=H L^{2}$ and that the surface area, $\mathrm{S}$, is given by $S=4 H L+2 L^{2}$, and that the assumptions under which the mean Dirac chord length are valid [15], such that $r=4 \mathrm{~V} / \mathrm{S}$, then (A.5) is equivalent to (6), considering that $\Sigma_{A}=(1 / V) \times \sum_{i} \frac{m_{i}}{A_{i}}\left(\sigma_{f}(t)+\sigma_{c}(t)\right)_{i}$.

From this analysis, it is evident that the current analytical $f(t)$ given by (6) in section 2.0 is based on a number of assumptions. From instance, it is based on the conditions of the interrogation neutron flux at the detectors to being equal to the neutron flux within the fuel volume for the case when the interrogation chamber contains a material (e.g. air) for which neutron interactions can be assumed to be negligible. The analytical $f(t)$ assumes that the flux within the fuel volume is uniform and isotropic. A comparison between (A.5) and (6) is shown in Figure A.1, along with the true $f(t)$ calculated directly from MCNPX for the case of $30 \mathrm{GWd} / \mathrm{MTU}, 3 \%$ enriched, 1 year cooled fuel assembly. As shown, both of the analytical $f(t)$ 's are quite similar over a significant portion of the continuously slowing-down time region of interest. This indicates that there is no significant advantage for the use of the more general equation given by (A.5) over that given by 
(6), except that multiplication by 0.8 , the $f(t)$ for the empty interrogation chamber, is not required for (A.5).

The value of $4 V / S$ for the mean chord length is based on the assumptions of uniform and isotropic flow of particles (e.g. neutrons) into the volume of interest (e.g. fuel assembly parallelepiped) and no absorption or particle production in the medium [15]. It should be noted that the $4 \mathrm{~V} / \mathrm{S}$ result holds true for a wide range of situations for which scattering centers are present in the volume of interest [15]. This value of $4 \mathrm{~V} / \mathrm{S}$ is equal to $19.4 \mathrm{~cm}$ for the dimensions of the fuel assembly model used in this study. This theoretical value was tested in MCNPX with two separate cases. The first test case simulated consisted of the PNNL LSDS model with the fuel assembly volume filled completely with air, and the second test case was for the fuel assembly volume filled entirely of ${ }^{238} \mathrm{U}$. Only interrogation neutrons having energies below $1 \mathrm{keV}$ were considered, so a test case using fissile material was not used, since the energies of fission neutrons are generally on the order of MeV. A user-supplied subroutine (TallyX) was implemented in MCNPX to calculate the path of each neutron of energy below $1 \mathrm{keV}$ through the fuel assembly volume, for neutron slowing-down times between $20 \mu \mathrm{s}$ and $2000 \mu \mathrm{s}$, assuming its path were unobstructed by absorption, scattering, etc. Interestingly, the value of the mean chord length obtained for each of the two test cases were very close to the theoretical value of $19.4 \mathrm{~cm}$, with the calculated value being $19.5 \mathrm{~cm}$ for the air-filled case and $19.7 \mathrm{~cm}$ for the ${ }^{238} \mathrm{U}$-filled case. Therefore, it was concluded that the theoretical value of the chord length (i.e. $4 V / S)$ used in the derivation of (6) is valid.

The current analytical $f(t)$ also assumes negligible scattering, in addition to the neutron-absorbing materials in the fuel being distributed homogeneously throughout the fuel volume, as required by (A.1) and (A.2). In fact, working strictly within the mathematical framework given by (A.5), the only presently conceivable way that the effects of streaming and scattering can be included in the analytical model is, in (A.5), to replace $r$ by the average neutron track length, $k$, (i.e. the average distance a neutron travels before being scattered, absorbed, etc.) and $N$ by the total number of neutron tracks, $T$, within the fuel volume. The quantities $k$ and $T$ were determined in MCNPX using TallyX subroutines. The result is shown in Figure A.2 for the $30 \mathrm{GWd} / \mathrm{MTU}, 3 \%$ enriched, 1 year cooled fuel assembly. As shown in Figure A.2., this analytical form for $f(t)$ matches very closely the true $f(t)$ result obtained directly from MCNPX by dividing the tallied flux in the fuel by the tallied flux in the detectors. Although, if one is required to average neutron track length and the total number of neutron tracks within the fuel, one is, by definition, required to know $\bar{\phi}_{f u e l}(t)$, since $\bar{\phi}_{\text {fuel }}(t)$ is simply the product of these two quantities normalized by the volume occupied by the fuel. 


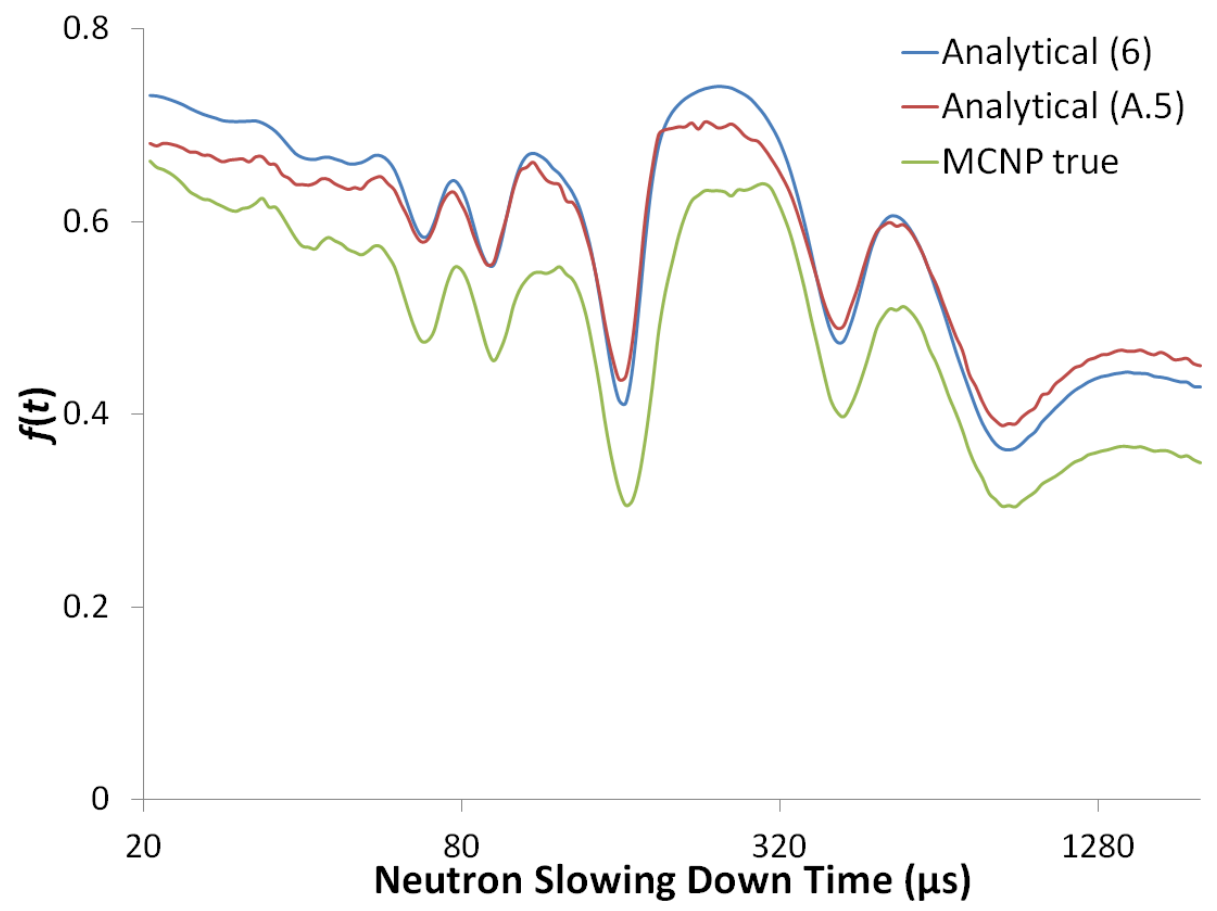

Figure A.1. Comparison between the $f(t)$ obtained by dividing the actual average flux in the fuel by the actual average flux in the surrounding detectors as tallied in the MCNP simulations [7] versus the analytical $f(t)$ calculated using (6) as well as (A.5). The results shown are for a 30 GWd/MTU, 3\% enriched, 1yr cooled used fuel assembly. 
PNNL-20769

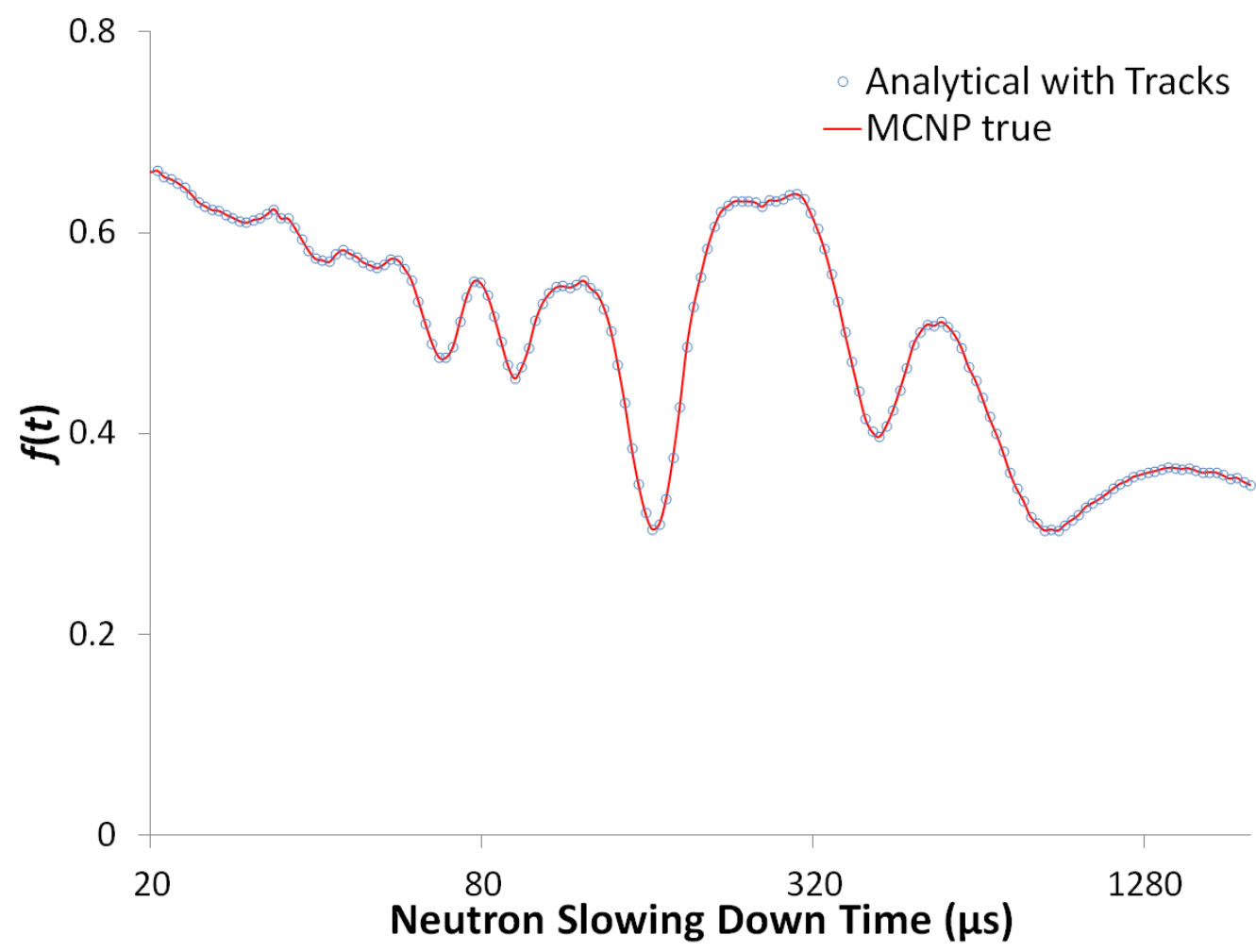

Figure A.2. The true $f(t)$ shown in this figure was determined in MCNPX by dividing the tallied flux in the fuel by the average tallied flux in the detectors. The analytical estimate in this figure was calculated using a modified version of (A.5), obtained by replacing $r$ by $k$ and $N$ by $T$ in (A.5), where $k$ and $T$ are defined in the text. The results shown are for a $30 \mathrm{GWd} / \mathrm{MTU}, 3 \%$ enriched, 1yr cooled used fuel assembly. 


\section{Appendix B: Numerical Methods and Fitting Algorithms}

All of the time-spectral analysis algorithms described in this paper incorporated the Poisson variability that would be expected in real data. Poisson statistical counting uncertainty was simulated into the assay signal, $y(t)$, and the isotopic response functions, $x_{i}(t)$. For the assay signal, Poisson counts were simulated in each time bin, $Y(t)$, with expected number of counts equal to $y(t)^{*} S F y^{*} M^{*} N$, where $S F y$ is a scaling factor equal to 0.001 to account for the absolute detection efficiency of 32 threshold fission chambers (sizes specified in [7]), each lined with a 1micron layer of active materials (e.g. ${ }^{232} \mathrm{Th}$ or ${ }^{238} \mathrm{U}$ ), $M$ is the total number of neutrons emitted per pulse, and $N$ is the number of pulses utilized in the simulated assay (e.g. one pulse every 10 milliseconds for a 15 -minute assay would result in approximately $1 \times 10^{5}$ pulses). For the isotopic response functions, the simulated Poisson counts, $X_{i}(t)$ used an expected number of counts equal to $x_{i}(t)^{*} S F x^{*} M^{*} N$, where $S F x$ is a scaling factor equal to 0.0025 to account for the absolute detection efficiency of a single fission chamber lined with an active layer of 1 micron of ${ }^{239} \mathrm{Pu}$, ${ }^{241} \mathrm{Pu}$ or ${ }^{235} \mathrm{U}$, also with the fission chamber size described in [7]. For all of the analysis presented in this report, the $M$ and $N$ values were set at $10^{8}$, resulting in a total number of emitted neutrons of $10^{16}$ for the assay of each assembly, the estimated required number of source neutrons, as described in section 4.0 of this report.

The fitting method used for in this work, the Maximum Likelihood Estimation (MLE), involves minimizing the objective function, $R$, (the negative log-likelihood):

$$
R=\sum_{j=1}^{n}\left(y^{\prime}\left(t_{j}\right)-Y\left(t_{j}\right) \log \left(y^{\prime}\left(t_{j}\right)\right)\right)
$$

where $\log$ is the natural logarithm and $y^{\prime}\left(t_{j}\right)$ is the calculated assay signal using (B.2):

$$
y^{\prime}\left(t_{j}\right)=f\left(t_{j}\right) C \sum_{i} \frac{m_{i}}{\bar{v}_{i} A_{i}} X_{i}\left(t_{j}\right)
$$

In (B.2), (6) was substituted for $f\left(t_{j}\right)$ when using the pure analytical approach, (8) was substituted for $f\left(t_{j}\right)$ when using the pure empirical approach, and (B.3) was substituted for $f\left(t_{j}\right)$ when using the analytical approach: 


$$
f(t)=\left[\frac{1-e^{-\left(\frac{2 N_{A}}{L(L+2 H)} \sum_{i} \frac{m_{i}}{A_{i}}\left(\sigma_{f}(t)+\sigma_{c}(t)\right)_{i}\right)}}{\frac{2 N_{A}}{L(L+2 H)} \sum_{i} \frac{m_{i}}{A_{i}}\left(\sigma_{f}(t)+\sigma_{c}(t)\right)_{i}}\right] \times\left[1.0+\sum_{j=1}^{5} b_{j} B_{j}(t)\right]
$$

The masses of ${ }^{235} \mathrm{U},{ }^{239} \mathrm{Pu},{ }^{240} \mathrm{Pu}$, and ${ }^{241} \mathrm{Pu}$ were substituted for the $m_{i}$ 's in (B.3).

The MLE parameter estimates are the values of the $m_{i}$ 's (for the pure analytical approach), or the $m_{i}$ 's and $b_{j}$ 's (for the pure empirical or hybrid analytical-empirical approach), that minimize the objective function $R$ in (B.1) subject to non-negativity constraints on the $m_{i}$ 's. 


\subsection{References}

1. N. Peter, "A Shipper-Receiver-Difference Approach for Reprocessing Plants," 8th International Conference on Facility Operations - Safeguards Interface, Portland OR, 2008.

2. Y. Danon, et al, "Measurements with the high flux lead slowing-down spectrometer at LANL," Nuclear Instruments and Methods in Physics Research B, 261/1-2, pp. 953-955, 2007.

3. C. Romano, et al, "Measurements of $(n, \alpha)$ cross-section of small samples using a leadslowing-down-spectrometer," Nuclear Instruments and Methods in Physics Research A, 562/2, pp. 771-773, 2006.

4. L.E. Smith and N.M. Abdurrahman, "Neutron Spectrometry for the Assay of High Fissile Content Spent Fuel," Nucl. Tech. 140, 328 (2002).

5. J.J. Ressler, L.E. Smith, and K .K. Anderson, "Lead Slowing Down Spectroscopy for Direct Pu Mass Measurements." in 8th International Conference on Facility Operations Safeguards Interface, Portland OR (2008).

6. L.E. Smith, KK Anderson, J.J. Ressler, S.D. Kiff, M.W. Shaver, "Time-Spectral Analysis Algorithms for Lead Slowing-Down Spectroscopy of Spent Fuel," 2009 Institute of Nuclear Materials Management Conference, Tucson, AZ, July 2009.

7. L.E. Smith, C.J. Gesh, K.K. Anderson, A.M.Casella, and M.W. Shaver, "Lead SlowingDown Spectroscopy for Direct Measurement of Plutonium in Spent Fuel: NGSI Phase I Report. PNNL-20158, Pacific Northwest National Laboratory, Richland, WA. 2010.

8. S.J. Tobin, et al., "Determination of Plutonium Content in Spent Fuel with Nondestructive Assay," 2009 Institute of Nuclear Materials Management Conference, Tucson, AZ, 2009.

9. D.B. Pelowitz, “MCNPX User's Manual”, Vol. LA-CP-07-1473 Version 2.60, Department of Energy, Los Alamos National Laboratory, Los Alamos, NM: U.S., 2008.

10. J.A. Kulisek, et al, "Progress on Establishing the Feasibility of Lead Slowing Down Spectroscopy for Direct Measurement of Plutonium in Used Fuel," 2011 Institute of Nuclear Materials Management Conference, Palm Desert, CA, July 2011. 
11. Y.D. Lee, et al. "Design of a Spent-Fuel Assay Device Using a Lead Spectrometer," Nucl. Sci. Eng. 131/1, pp. 45-61, 1999.

12. P. A. M. Dirac, Approximate Rate of Neutron Multiplication for a Solid of Arbitrary Shape and Uniform Density, Declassified British Report MS-D-5, Part I, 1943.

13. G. H. Golub and C. Reinsch, "Singular Values and Least Squares Solutions," Numer. Math. 14, pp. 403-420, 1970.

14. A. Gavron, R.L. Haight, M. Devlin, and J.M. O’Donnell, "Predicted Precision and Accuracy of LSDS Spent Fuel Measurement," LA-UR-11-04626, Los Alamos National Laboratory, Los Alamos, NM. 2011.

15. J.N. Bardsley and A. Dubi, "The Average Transport Path Length in Scattering Media," SIAM J. Appl. Math. 40, No. 1, pp. 71-77, February 1981. 


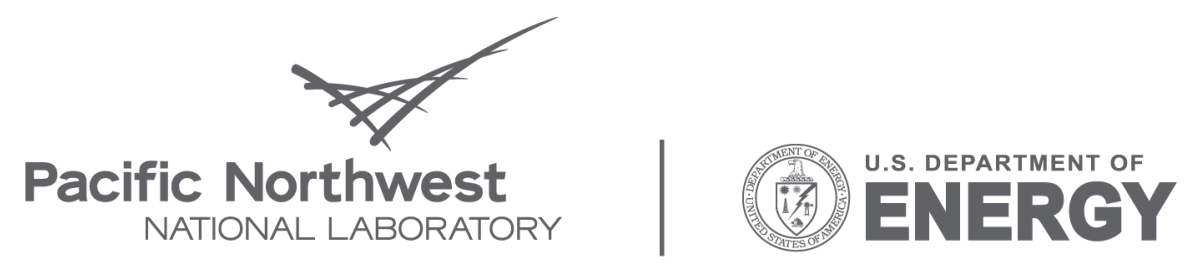

Proudly Operated by Battelle Since 1965

902 Battelle Boulevard

P.O. Box 999

Richland, WA 99352

1-888-375-PNNL (7665)

www.pnl.gov 